\title{
Levantamento de espécies de cobertura vegetal nativas e exóticas encontradas no campus oeste da Universidade Federal Rural do Semi-Árido
}

\section{Survey of species of native and exotic vegetation coverage found in the campus of Federal Rural University west of the Semi-arid region}

\author{
Leonardo Vieira de Sousa ${ }^{1}$, Idalice Emilly de Oliveira Teixeira ${ }^{2}$, Maria Gabriela Fernandes Martins de Medeiros ${ }^{3}$, André \\ Luiz da Silva ${ }^{4}$, Francisco de Assis de Oliveira ${ }^{5}$
}

\begin{abstract}
Resumo: Desmatamento é o processo de desaparecimento de massas florestais, fundamentalmente causadas pela atividade humana, com a retirada da cobertura vegetal em determinada área para utilização comercial de madeira, implantação de projetos agropecuários e também na expansão urbana. Como em qualquer área que necessite de uma cobertura vegetal, a UFERSA também enfrenta uma problemática frequente, a replantação de árvores exóticas invasoras, ao invés de nativas da região. Dessa forma o presente trabalho tem como objetivo não somente avaliar e mapear a densidade da flora do local, mas também evidenciar possíveis problemas e disponibilizar soluções. Procurou-se dividir a vegetação em dois grupos: as nativas e as exóticas. Foi realizada a demarcação dos pontos de acordo com a localização das espécies, com a finalidade de identificar e quantificar as espécies de plantas nativas e exóticas. Dentre todas as espécies amostradas, 24 espécies (25,3\%) são representantes de espécies nativas e 71 espécies (74,7\%) são exóticas. A arborização da UFERSA segue o padrão observado na maioria das áreas verdes públicas das cidades brasileiras, com grande número de espécies exóticas em relação às nativas. Em vista de uma melhor estrutura física fica evidente a necessidade de uma maior arborização, tendo como incentivo o plantio de espécies nativas, que proporcionem sombra, alimento e sirvam de abrigo para a fauna local, não apenas na UFERSA, mas em toda a região que vem perdendo sua flora natural em favor de plantas exóticas.
\end{abstract}

Palavras-chaves: UFERSA, vegetação, impacto ambiental, desmatamento, reflorestamento.

\begin{abstract}
Deforestation is the process of disappearance of forest masses, basically caused by human activity, with the withdrawal of the vegetation cover in particular area for commercial use of wood, deployment of agricultural projects and also in urban expansion. As in any area that needs a plant cover, the UFERSA also faces a frequent problem, replanting trees invasive alien, instead of native to the region. This way the objective of the present work is to not only assess and map the density of the flora of the site but also highlight potential problems and provide solutions. We sought to divide the vegetation in two groups: the native and exotic. Was performed the demarcation of points of agreement with the location of the species, with the purpose of identifying and quantifying the species of native and exotic plants. Among all the species sampled, 24 species $(25.3 \%)$ are representatives of native species and 71 species $(74.7 \%)$ are exotic. The afforestation of UFERSA follows the pattern observed in the majority of public green areas of the Brazilian cities with large number of exotic species in relation to the native. In view of a better physical structure, it is evident the need for a greater afforestation, having as encouraging the planting of native species, which provide shade, food and serve as a shelter for the local fauna, not only in UFERSA, but throughout the region that is losing its natural flora in favor of exotic plants.
\end{abstract}

Key words: UFERSA, vegetation, environmental impact, deforestation, reforestation.

\footnotetext{
*Autor para correspondência

Recebido para publicação em 10/11/2015; aprovado em 22/12/2015

${ }^{1}$ Graduando em Agronomia, Universidade Federal Rural do Semi-Árido, Mossoró-RN; (84) 998308279, E-mail: leoigt@ hotmail.com.

${ }^{2}$ Graduando em Ecologia, Universidade Federal Rural do Semi-Árido, E-mail: licemilly@ hotmail.com

${ }^{3}$ Graduando em Ecologia, Universidade Federal Rural do Semi-Árido, E-mail: mabi_life@ hotmail.com

${ }^{4}$ Graduando em Ecologia, Universidade Federal Rural do Semi-Árido, E-mail: andluizds@ @mail.com

${ }^{5}$ Doutor em Irrigação e Drenagem pela Escola Superior de Agricultura Luiz de Queiroz, prof. Adjunto da UFERSA, E-mail: thikaoamigao@ufersa.edu.br
} 


\section{INTRODUÇÃO}

Desflorestação ou desmatamento é o processo de desaparecimento de massas florestais, fundamentalmente causadas pela atividade humana, com a retirada da cobertura vegetal em determinada área para utilização comercial de madeira, implantação de projetos agropecuários e também na expansão urbana (SILVA, 2006).

O desmatamento da floresta amazônica brasileira consiste em um dos principais problemas enfretados pelo Brasil atualmente. Os dados divulgados pelo instituto nacional de pesquisas espaciais (INPE, 2014) sobre a retirada da cobertura florestal na região atestam que, nos últimos dez

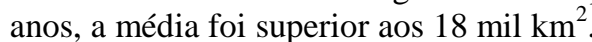

Para tentar contornar esses problemas ambientais existe a possibilidade de reflorestamento, aumentando o número de árvores e com isso promovendo o sequestro de $\mathrm{CO}_{2}$ da atmosfera, diminuindo assim a concentração deste gás e consequentemente, desempenhando um importante papel no combate á intensificação do efeito estufa. A remoção do gás carbônico da atmosfera é realizada graças á fotossíntese, permitindo a fixação do carbono na biomassa da vegetação e nos solos.

Como em qualquer área que necessite de uma cobertura vegetal, a UFERSA também enfrenta uma problemática frequente, a replantação de árvores exóticas invasoras, ao invés de nativas da região, sem conhecimento prévio dos efeitos destas plantas para o novo ambiente.

Existem diversos fatores ecológicos para a escolha do replantio de árvores nativas á exóticas. Entre eles podemos citar que as árvores nativas estão bem adaptadas a região e que servem para a alimentação de herbívoros, além de guardar características ambientais da região. Já as exóticas por serem recém-chegadas ao ambiente, dificilmente terão predadores naturais, conferindo a possibilidade de multiplicação sem controle, podendo torna-se uma praga. Por não terem uma boa relação com a floresta nativa, podem competir desigualmente por água, luz e nutrientes, podendo até matar as espécies nativas.

Algumas espécies exóticas tem as raízes muito bem preparadas para absorver água, como é o caso do eucalipto, que absorve tanta água do solo, que este chega a ficar seco.

O maior erro em plantar árvores exóticas é que estas espécies crescem rapidamente. Pessoas e empresas que são obrigadas judicialmente a reflorestar utilizam estas espécies para mostrar resultados rapidamente (ÁRVORES BRASIL, 2015).

Dessa forma o presente trabalho tem como objetivo não somente avaliar e mapear a densidade da flora do local de interesse, mas também evidenciar possíveis problemas e disponibilizar soluções.

\section{MATERIAL E MÉTODOS}

O estudo foi realizado na Universidade Federal Rural do Semi-Árido, campus Mossoró-RN, situando-se a $5^{\circ} 11^{\prime}$ de latitude sul e $37^{\circ} 20^{\prime}$ de longitude oeste e altitude de $18 \mathrm{~m}$, o município localiza-se na mesorregião do oeste potiguar, distante da capital Natal $278 \mathrm{~km}$, possuindo uma população estimada em 259.886 habitantes com uma área de 2.099,33 $\mathrm{km}^{2}$ (IBGE, 2010).
Segundo Thornthwaite, o clima do local é DdAa, ou seja, semiárido, megatérmico e com pequeno ou nenhum excesso de água durante o ano, e de acordo com Koppen é BSwh, seco e muito quente, com duas estações climáticas: uma seca, que geralmente compreende o período de junho a janeiro e outra chuvosa, entre os meses de fevereiro a maio (CARMO FILHO et al., 1991).

Procurou-se dividir a vegetação em dois grupos: as nativas e as exóticas.

Foi realizada a demarcação dos pontos de acordo com a localização das espécies vegetais de interesse, com a finalidade de identificar e quantificar as espécies de plantas nativas e exóticas tendo como ponto de estudo a flora do bioma caatinga.

O levantamento foi realizado em fevereiro de 2014 e os dados foram coletados com o auxilio de um GPS (Sistema de Posicionamento Global) portátil GarminEtrex 10 trilha Glonass, programado em UTM (Universal Transversa de Mercator) que utiliza um sistema de coordenadas cartesianas bidimensional para dar localizações na superfície da terra.

Após a coleta, os dados foram transferidos para o computador com o auxílio do programa TrackMaker, comumente empregado para a criação de mapas detalhados. Logo após os pontos foram quantificados e sincronizados com o mapa da área estudada.

\section{RESULTADOS E DISCUSSÃO}

No presente levantamento encontram-se um total de 24 plantas sendo representadas por 9 espécies nativas da região (Tabela 1), com destaque para o Mandacaru (Cereus jamacaru) a Carnaubeira (Copernicia prunifera) e a Favela (Cnidoscolus quercifolius), sendo estes os principais exemplos de espécies nativas de maior incidência no bioma caatinga, destacando-se como as principais plantas encontradas durante a demarcação dos pontos da área que realizou-se o estudo.

A espécie nativa que obteve maior número de indivíduos foi a Copernicia prunifera, com cinco plantas, representando um total de $21 \%$ das espécies nativas estudadas.

As espécies nativas são importantes economicamente para a região se destacando na utilização para alimentação animal como também com a finalidade de sombrear o ambiente e preservar as características vegetais da região (CORADIN, 2011).

A Carnaubeira é uma árvore endêmica do semi-árido da região Nordeste do Brasil, sendo adaptada ao clima da região. A carnaúba oferece possibilidades de atividades econômicas mesmo durante o período de estiagem, tratando-se, portanto de importante alternativa na composição da renda familiar das comunidades rurais (BARBOSA, 2009).

Os carnaubais formam florestas que tem predominâncias nas planícies aluviais dos principais rios do Ceará, Piauí, Maranhão, Rio Grande do Norte e Bahia, cumprindo importantes funções para a manutenção do equilíbrio ecológico da região, como a conservação dos solos, fauna, cursos d'água e mananciais hídricos. 
Tabela 1 - Coordenadas geográficas de espécies vegetais nativas encontradas no campus oeste da Universidade Federal Rural do Semi-Árido (UFERSA). Mossoró- RN, 2014.

\begin{tabular}{|c|c|c|c|c|}
\hline \multirow{2}{*}{ Ponto } & \multirow{2}{*}{$\begin{array}{c}\text { Nome } \\
\text { Científico }\end{array}$} & \multirow{2}{*}{ Nome Vulgar } & \multicolumn{2}{|c|}{ Coordenadas } \\
\hline & & & Latitude & Longitude \\
\hline 1 & Cereus jamacaru & Mandacaru & $5^{\circ} 12^{\prime} 0.17^{\prime \prime} \mathrm{S}$ & $37^{\circ} 19^{\prime} 00.47^{\prime \prime} \mathrm{O}$ \\
\hline 2 & Cnidoscolus quercifolius & Favela & $5^{\circ} 12^{\prime} 1.71 ' ’ S$ & $37^{\circ} 19^{\prime} 09.32^{\prime \prime} \mathrm{O}$ \\
\hline 3 & Ziziphus jozeiro Mart. & Juazeiro & $5^{\circ} 12^{\prime} 0.18^{\prime \prime} \mathrm{S}$ & $37^{\circ} 19^{\prime} 09.83^{\prime \prime} \mathrm{O}$ \\
\hline 4 & Bixa orellana & Urucuzeiro & $5^{\circ} 12^{\prime} 0.19^{\prime \prime} \mathrm{S}$ & $37^{\circ} 19^{\prime} 03.73^{\prime \prime} \mathrm{O}$ \\
\hline 5 & Copernicia prunifera & Carnaubeira & $5^{\circ} 12^{\prime} 0.16^{\prime \prime} \mathrm{S}$ & $37^{\circ} 19^{\prime} 87.46^{\prime \prime} \mathrm{O}$ \\
\hline 6 & Cereus jamacaru & Mandacaru & $5^{\circ} 12^{\prime} 0.18^{\prime \prime} \mathrm{S}$ & $37^{\circ} 19^{\prime} 94.74^{\prime \prime} \mathrm{O}$ \\
\hline 7 & Cnidoscolus quercifolius & Favela & $5^{\circ} 12^{\prime} 0.21^{\prime \prime} \mathrm{S}$ & $37^{\circ} 19^{\prime} 93.78^{\prime \prime} \mathrm{O}$ \\
\hline 8 & Cereus jamacaru & Mandacaru & $5^{\circ} 12^{\prime} 0.19^{\prime \prime} \mathrm{S}$ & $37^{\circ} 19^{\prime} 90.30^{\prime \prime} \mathrm{O}$ \\
\hline 9 & Copernicia prunifera & Carnaubeira & $5^{\circ} 12^{\prime} 0.28^{\prime \prime} \mathrm{S}$ & $37^{\circ} 19^{\prime} 09.97^{\prime \prime} \mathrm{O}$ \\
\hline 10 & Copernicia prunifera & Carnaubeira & $5^{\circ} 12^{\prime} 0.25^{\prime \prime} \mathrm{S}$ & $37^{\circ} 32^{\prime} 87.53^{\prime \prime} \mathrm{O}$ \\
\hline 11 & Copernicia prunifera & Carnaubeira & $5^{\circ} 12^{\prime} 0.20{ }^{\prime \prime} \mathrm{S}$ & $37^{\circ} 19^{\prime} 65.34^{\prime \prime} \mathrm{O}$ \\
\hline 12 & Ziziphus jozeiro Mart. & Juazeiro & $5^{\circ} 12^{\prime} 0.36^{\prime \prime} \mathrm{S}$ & $37^{\circ} 19^{\prime} 98.71^{\prime \prime} \mathrm{O}$ \\
\hline 13 & Ziziphus jozeiro Mart. & Juazeiro & $5^{\circ} 12^{\prime} 2.20{ }^{\prime} \mathrm{S}$ & $37^{\circ} 19^{\prime} 85.65^{\prime \prime} \mathrm{O}$ \\
\hline 14 & Cnidoscolus quercifolius & Favela & $5^{\circ} 12^{\prime} 0.26^{\prime \prime} \mathrm{S}$ & $37^{\circ} 19^{\prime} 36.53^{\prime \prime} \mathrm{O}$ \\
\hline 15 & Tabeluia heptaphylla & Ipê-Rosa & $5^{\circ} 12^{\prime} 0.27^{\prime \prime} \mathrm{S}$ & $37^{\circ} 19^{\prime} 38.73^{\prime \prime} \mathrm{O}$ \\
\hline 16 & Tabeluia avellanedae & Ipê-Roxo & $5^{\circ} 12^{\prime} 2.50 ’ ’ \mathrm{~S}$ & $37^{\circ} 19^{\prime} 44.04^{\prime \prime} \mathrm{O}$ \\
\hline 17 & Tabeluia áurea & Ipê-Amarelo & $5^{\circ} 12^{\prime} 0.27^{\prime \prime} \mathrm{S}$ & $37^{\circ} 19^{\prime} 94.86^{\prime \prime} \mathrm{O}$ \\
\hline 18 & Copernicia prunifera & Carnaubeira & $5^{\circ} 12^{\prime} 0.25^{\prime \prime} \mathrm{S}$ & $37^{\circ} 19^{\prime} 73.36^{\prime \prime} \mathrm{O}$ \\
\hline 19 & Erythrina mulungu & Mulungu & $5^{\circ} 12^{\prime} 0.32{ }^{\prime} \mathrm{S}$ & $37^{\circ} 19^{\prime} 21.41^{\prime \prime} \mathrm{O}$ \\
\hline 20 & Cnidoscolus quercifolius & Favela & $5^{\circ} 12^{\prime} 0.28^{\prime \prime} \mathrm{S}$ & $37^{\circ} 19^{\prime} 25.25^{\prime \prime} \mathrm{O}$ \\
\hline 21 & Erythrina mulungu & Mulungu & $5^{\circ} 12^{\prime} 0.29^{\prime \prime} \mathrm{S}$ & $37^{\circ} 19^{\prime} 08.28^{\prime \prime} \mathrm{O}$ \\
\hline 22 & Cereus jamacaru & Mandacaru & $5^{\circ} 12^{\prime} 0.31^{\prime \prime} \mathrm{S}$ & $37^{\circ} 19^{\prime} 92.38^{\prime \prime} \mathrm{O}$ \\
\hline 23 & Tabeluia avellanedae & Ipê-Roxo & $5^{\circ} 12^{\prime} 0.31^{\prime \prime} \mathrm{S}$ & $37^{\circ} 19^{\prime} 01.98^{\prime \prime} \mathrm{O}$ \\
\hline 24 & Erythrina mulungu & Mulungu & $5^{\circ} 12^{\prime} 0.34{ }^{\prime \prime} \mathrm{S}$ & $37^{\circ} 19^{\prime} 02.99^{\prime \prime} \mathrm{O}$ \\
\hline
\end{tabular}

Além das espécies anteriormente citadas, também foram mapeadas as espécies exóticas da área (Tabela 2). Foram encontradas 71 plantas arbóreas representadas por 19 espécies, com maior ocorrência de neem (Azadirachta indica) e mangueira (Mangifera indica).

A espécie presente em maior número é a Azadirachta indica, conhecida popularmente como neem que representa $35 \%$ (33 indivíduos) do total das espécies estudadas. Essa espécie encontra-se hoje em quase todas as regiões do Brasil devido principalmente ser uma planta arbórea de crescimento rápido e adaptada às condições climáticas do país.

Segundo Randall (2002), o neem compete com outras espécies arbóreas em ambientes florestais e domina pela ação de substâncias alelopáticas, inibindo a germinação de espécies nativas. Altera também o regime hídrico, principalmente em ecossistemas abertos, onde substitui a vegetação de pequeno porte. Provoca perda de biodiversidade por sombreamento, provocando exposição do solo e, consequente, erosão e assoreamento de cursos d'água.

Já a Mangifera indica foi a segunda espécie de maior incidência, representando 13\% (12 indivíduos) das plantas estudadas.

A invasão dessa espécie em ambientes ciliares pode provocar alteração do $\mathrm{pH}$ da água devido ao apodrecimento das folhas e dos frutos em grande quantidade, também provoca impacto sobre a dispersão de espécies nativas zoocóricas, uma vez que é muito utilizada como fonte de alimento por animais, diminuindo o consumo de frutos e, consequentemente, a dispersão de espécies nativas (GISP, 2005).

A mangueira é uma das principais plantas exóticas invasoras encontradas no semiárido Nordestino. É também invasora m diversos países como: México, Austrália, China, Estados Unidos (Havaí), Equador (Ilhas Galápagos), Fiji, Polinésia Francesa, Guam, Japão, Nauru, Nova Caledônia, Niue, França (Ilhas Reunião), Ilhas Maurício, em Tonga e no Paquistão (OLIVEIRA; ZAKIA, 2010).

Com relação às demais espécies exóticas encontradas na UFERSA, estas tiveram pouca representatividade em comparação ao neem e a mangueira.

Ainda que nem todas as espécies exóticas observadas apresentem comportamento invasor no local estudado, a grande maioria tem potencial de invasão em função de seu histórico em outros locais, já que algumas constam na base de dados nacional de espécies exóticas invasoras (INSTITUTO HÓRUS, 2007). 
Leonardo Vieira de Sousa et al.

Tabela 2 - Coordenadas geográficas de espécies vegetais exóticas encontradas no campus oeste da Universidade Federal Rural do Semi-Árido (UFERSA). Mossoró- RN, 2014.

\begin{tabular}{|c|c|c|c|c|}
\hline \multirow{2}{*}{ Ponto } & \multirow{2}{*}{$\begin{array}{c}\text { Nome } \\
\text { Científico }\end{array}$} & \multirow{2}{*}{ Nome Vulgar } & \multicolumn{2}{|c|}{ Coordenadas } \\
\hline & & & Latitude & Longitude \\
\hline 1 & Azadirachta indica & Neem & $5^{\circ} 12^{\prime} 1.53^{\prime \prime} \mathrm{S}$ & $37^{\circ} 19^{\prime} 77.21^{\prime \prime} \mathrm{O}$ \\
\hline 2 & Phoenix canariensis & Palmeira & $5^{\circ} 12^{\prime} 1.61 ' ’ \mathrm{~S}$ & $37^{\circ} 19^{\prime} 77.38^{\prime \prime} \mathrm{O}$ \\
\hline 3 & Azadirachta indica & Neem & $5^{\circ} 12^{\prime} 1.65^{\prime \prime} \mathrm{S}$ & $37^{\circ} 19^{\prime} 77.24^{\prime \prime} \mathrm{O}$ \\
\hline 4 & Malpighia glabra L. & Acerola & $5^{\circ} 12 ’ 1.68 ’ \mathrm{~S}$ & $37^{\circ} 19^{\prime} 76.90^{\prime \prime} \mathrm{O}$ \\
\hline 5 & Acacia heterophylla & Acace & $5^{\circ} 12{ }^{\prime} 1.69$ '’ $\mathrm{S}$ & $37^{\circ} 19^{\prime} 76.87^{\prime \prime} \mathrm{O}$ \\
\hline 6 & Terminalia catappa & Castanhola & $5^{\circ} 12^{\prime} 1.82{ }^{\prime \prime} \mathrm{S}$ & $37^{\circ} 19^{\prime} 75.29^{\prime \prime} \mathrm{O}$ \\
\hline 7 & Azadirachta indica & Neem & $5^{\circ} 12^{\prime} 1.72, ' \mathrm{~S}$ & $37^{\circ} 19^{\prime} 73.96^{\prime \prime} \mathrm{O}$ \\
\hline 8 & Tamarindus indica $\mathrm{L}$. & Tamarindo & $5^{\circ} 12^{\prime} 1.92 ’ ’ \mathrm{~S}$ & $37^{\circ} 19^{\prime} 73.66^{\prime \prime} \mathrm{O}$ \\
\hline 9 & Eucalyptus globulus & Eucalipto & $5^{\circ} 12^{\prime} 2.07^{\prime \prime} \mathrm{S}$ & $37^{\circ} 19^{\prime} 71.73^{\prime \prime} \mathrm{O}$ \\
\hline 10 & Musa paradisíaca $\mathrm{L}$. & Bananeira & $5^{\circ} 12^{\prime} 2.16^{\prime \prime} \mathrm{S}$ & $37^{\circ} 19^{\prime} 73.10^{\prime \prime} \mathrm{O}$ \\
\hline 11 & Azadirachta indica & Neem & $5^{\circ} 12{ }^{\prime} 2.01 ' ’ \mathrm{~S}$ & $37^{\circ} 19^{\prime} 69.87^{\prime \prime} \mathrm{O}$ \\
\hline 12 & Azadirachta indica & Neem & $5^{\circ} 12^{\prime} 2.03{ }^{\prime \prime} \mathrm{S}$ & $37^{\circ} 19^{\prime} 68.90^{\prime \prime} \mathrm{O}$ \\
\hline 13 & Psidium guajava & Goiabeira & $5^{\circ} 12^{\prime} 2.24^{\prime \prime} \mathrm{S}$ & $37^{\circ} 19^{\prime} 71.88^{\prime \prime} \mathrm{O}$ \\
\hline 14 & Azadirachta indica & Neem & $5^{\circ} 12 ’ 2.36{ }^{\prime \prime} \mathrm{S}$ & $37^{\circ} 19^{\prime} 72.49^{\prime \prime} \mathrm{O}$ \\
\hline 15 & Carica papaya $\mathrm{L}$. & Mamoeiro & $5^{\circ} 12^{\prime} 2.53^{\prime \prime} \mathrm{S}$ & $37^{\circ} 19^{\prime} 75.52^{\prime \prime} \mathrm{O}$ \\
\hline 16 & Passiflora edulis & Maracujazeiro & $5^{\circ} 12^{\prime} 2.54^{\prime \prime} \mathrm{S}$ & $37^{\circ} 19^{\prime} 74.79^{\prime \prime} \mathrm{O}$ \\
\hline 17 & Tamarindus indica L. & Tamarindo & $5^{\circ} 12{ }^{\prime} 2.71 ’ ’ \mathrm{~S}$ & $37^{\circ} 19^{\prime} 84.65^{\prime \prime} \mathrm{O}$ \\
\hline 18 & Azadirachta indica & Neem & $5^{\circ} 12^{\prime} 2.87^{\prime \prime} \mathrm{S}$ & $37^{\circ} 19^{\prime} 81.43^{\prime \prime} \mathrm{O}$ \\
\hline 19 & Azadirachta indica & Neem & $5^{\circ} 12^{\prime} 3.11^{\prime \prime} \mathrm{S}$ & $37^{\circ} 19^{\prime} 85.96^{\prime \prime} \mathrm{O}$ \\
\hline 20 & Mangifera indica & Mangueira & $5^{\circ} 12^{\prime} 2.87^{\prime \prime} \mathrm{S}$ & $37^{\circ} 19^{\prime} 77.011^{\prime} \mathrm{O}$ \\
\hline 21 & Mangifera indica & Mangueira & $5^{\circ} 12^{\prime} 2.90{ }^{\prime \prime} \mathrm{S}$ & $37^{\circ} 19^{\prime} 78.13^{\prime \prime} \mathrm{O}$ \\
\hline 22 & Azadirachta indica & Neem & $5^{\circ} 12^{\prime} 2.91{ }^{\prime \prime} \mathrm{S}$ & $37^{\circ} 19^{\prime} 73.67^{\prime \prime} \mathrm{O}$ \\
\hline 23 & Azadirachta indica & Neem & $5^{\circ} 12^{\prime} 2.66^{\prime \prime} \mathrm{S}$ & $37^{\circ} 19^{\prime} 73.39^{\prime \prime} \mathrm{O}$ \\
\hline 24 & Tamarindus indica $\mathrm{L}$. & Tamarindo & $5^{\circ} 12^{\prime} 2.98^{\prime \prime} \mathrm{S}$ & $37^{\circ} 19^{\prime} 74.29^{\prime \prime} \mathrm{O}$ \\
\hline 25 & Mangifera indica & Mangueira & $5^{\circ} 12^{\prime} 3.04^{\prime \prime} \mathrm{S}$ & $37^{\circ} 19^{\prime} 72.15^{\prime \prime} \mathrm{O}$ \\
\hline 26 & Mangifera indica & Mangueira & $5^{\circ} 12^{\prime} 3.23{ }^{\prime \prime} \mathrm{S}$ & $37^{\circ} 19^{\prime} 74.48^{\prime \prime} \mathrm{O}$ \\
\hline 27 & Mangifera indica & Mangueira & $5^{\circ} 12^{\prime} 3.24{ }^{\prime \prime} \mathrm{S}$ & $37^{\circ} 19^{\prime} 85.64^{\prime \prime} \mathrm{O}$ \\
\hline 28 & Azadirachta indica & Neem & $5^{\circ} 12^{\prime} 3.34^{\prime \prime} \mathrm{S}$ & $37^{\circ} 19^{\prime} 86.34^{\prime \prime} \mathrm{O}$ \\
\hline 29 & Azadirachta indica & Neem & $5^{\circ} 12^{\prime} 3.41 ' \mathrm{~S}$ & $37^{\circ} 19^{\prime} 83.81^{\prime \prime} \mathrm{O}$ \\
\hline 30 & Mangifera indica & Mangueira & $5^{\circ} 12 ’ 3.50 ’ ' \mathrm{~S}$ & $37^{\circ} 19^{\prime} 76.67^{\prime \prime} \mathrm{O}$ \\
\hline 31 & Azadirachta indica & Neem & $5^{\circ} 12^{\prime} 3.74{ }^{\prime \prime} \mathrm{S}$ & $37^{\circ} 19^{\prime} 80.13^{\prime \prime} \mathrm{O}$ \\
\hline 32 & Mangifera indica & Mangueira & $5^{\circ} 12^{\prime} 3.87^{\prime \prime} \mathrm{S}$ & $37^{\circ} 19^{\prime} 85.53^{\prime \prime} \mathrm{O}$ \\
\hline 33 & Mangifera indica & Mangueira & $5^{\circ} 12^{\prime} 4.13^{\prime \prime} \mathrm{S}$ & $37^{\circ} 19^{\prime} 80.51^{\prime \prime} \mathrm{O}$ \\
\hline 34 & Azadirachta indica & Neem & $5^{\circ} 12^{\prime} 4.59^{\prime \prime} \mathrm{S}$ & $37^{\circ} 19^{\prime} 81.74^{\prime \prime} \mathrm{O}$ \\
\hline 35 & Azadirachta indica & Neem & $5^{\circ} 12^{\prime} 4.54^{\prime \prime} \mathrm{S}$ & $37^{\circ} 19^{\prime} 80.17^{\prime \prime} \mathrm{O}$ \\
\hline 36 & Mangifera indica & Mangueira & $5^{\circ} 12^{\prime} 4.30 ’ ’ \mathrm{~S}$ & $37^{\circ} 19^{\prime} 78.47^{\prime \prime} \mathrm{O}$ \\
\hline 37 & Azadirachta indica & Neem & $5^{\circ} 12^{\prime} 4.31 ' \mathrm{~S}$ & $37^{\circ} 19^{\prime} 77.36^{\prime \prime} \mathrm{O}$ \\
\hline 38 & Carica papaya $\mathrm{L}$. & Mamoeiro & $5^{\circ} 12^{\prime} 4.53^{\prime \prime} \mathrm{S}$ & $37^{\circ} 19^{\prime} 77.11^{\prime \prime} \mathrm{O}$ \\
\hline 39 & Azadirachta indica & Neem & $5^{\circ} 12^{\prime} 4.20^{\prime \prime} \mathrm{S}$ & $37^{\circ} 19^{\prime} 71.98^{\prime \prime} \mathrm{O}$ \\
\hline 40 & Spondia ssp. & Cajarana & $5^{\circ} 12^{\prime} 3.94 \prime \prime S$ & $37^{\circ} 19^{\prime} 70.84^{\prime \prime} \mathrm{O}$ \\
\hline 41 & Azadirachta indica & Neem & $5^{\circ} 12^{\prime} 3.71 ' \mathrm{~S}$ & $37^{\circ} 19^{\prime} 66.23^{\prime \prime} \mathrm{O}$ \\
\hline 42 & Spondia ssp. & Cajarana & $5^{\circ} 12^{\prime} 3.32^{\prime \prime} \mathrm{S}$ & $37^{\circ} 19^{\prime} 67.27^{\prime \prime} \mathrm{O}$ \\
\hline 43 & Azadirachta indica & Neem & $5^{\circ} 12^{\prime} 3.49^{\prime \prime} \mathrm{S}$ & $37^{\circ} 19^{\prime} 64.90^{\prime \prime} \mathrm{O}$ \\
\hline 44 & Azadirachta indica & Neem & $5^{\circ} 12^{\prime} 3.43^{\prime \prime} \mathrm{S}$ & $37^{\circ} 19^{\prime} 63.23^{\prime \prime} \mathrm{O}$ \\
\hline 45 & Mangifera indica & Mangueira & $5^{\circ} 12^{\prime} 3.08^{\prime \prime} \mathrm{S}$ & $37^{\circ} 19^{\prime} 70.76^{\prime \prime} \mathrm{O}$ \\
\hline 46 & Tamarindus indica $\mathrm{L}$. & Tamarindo & $5^{\circ} 12^{\prime} 2.95 ’ ’ \mathrm{~S}$ & $37^{\circ} 19^{\prime} 62.24^{\prime \prime} \mathrm{O}$ \\
\hline 47 & Spondia ssp. & Cajarana & $5^{\circ} 12^{\prime} 2.83^{\prime \prime} \mathrm{S}$ & $37^{\circ} 19^{\prime} 61.14^{\prime \prime} \mathrm{O}$ \\
\hline
\end{tabular}




\begin{tabular}{|c|c|c|c|c|}
\hline 48 & Phoenix canariensis & Palmeira & $5^{\circ} 12^{\prime} 2.70^{\prime \prime} \mathrm{S}$ & $37^{\circ} 19^{\prime} 63.16^{\prime \prime} \mathrm{O}$ \\
\hline 49 & Mangifera indica & Mangueira & $5^{\circ} 12^{\prime} 2.67^{\prime \prime} \mathrm{S}$ & $37^{\circ} 19^{\prime} 63.58^{\prime \prime} \mathrm{O}$ \\
\hline 50 & Azadirachta indica & Neem & $5^{\circ} 12^{\prime} 2.24^{\prime \prime} \mathrm{S}$ & $37^{\circ} 19^{\prime} 65.57^{\prime \prime} \mathrm{O}$ \\
\hline 51 & Azadirachta indica & Neem & $5^{\circ} 12^{\prime} 2.15^{\prime \prime} \mathrm{S}$ & $37^{\circ} 19^{\prime} 67.24^{\prime \prime} \mathrm{O}$ \\
\hline 52 & Azadirachta indica & Neem & $5^{\circ} 12^{\prime} 1.15^{\prime \prime} \mathrm{S}$ & $37^{\circ} 19^{\prime} 57.03^{\prime \prime} \mathrm{O}$ \\
\hline 53 & Azadirachta indica & Neem & $5^{\circ} 12 ’ 1.19$ '’ $\mathrm{S}$ & $37^{\circ} 19^{\prime} 62.79^{\prime \prime} \mathrm{O}$ \\
\hline 54 & Azadirachta indica & Neem & $5^{\circ} 12^{\prime} 1.26^{\prime \prime} \mathrm{S}$ & $37^{\circ} 19^{\prime} 64.47^{\prime \prime} \mathrm{O}$ \\
\hline 55 & Azadirachta indica & Neem & $5^{\circ} 12^{\prime} 1.26^{\prime \prime} \mathrm{S}$ & $37^{\circ} 19^{\prime} 64.47^{\prime \prime} \mathrm{O}$ \\
\hline 56 & Azadirachta indica & Neem & $5^{\circ} 12^{\prime} 1.39^{\prime \prime} \mathrm{S}$ & $37^{\circ} 19^{\prime} 64.83^{\prime \prime} \mathrm{O}$ \\
\hline 57 & Citrus limon & Limoeiro & $5^{\circ} 12^{\prime} 1.47^{\prime \prime} \mathrm{S}$ & $37^{\circ} 19^{\prime} 62.32^{\prime \prime} \mathrm{O}$ \\
\hline 58 & Azadirachta indica & Neem & $5^{\circ} 12^{\prime} 1.58^{\prime \prime} \mathrm{S}$ & $37^{\circ} 19^{\prime} 64.85^{\prime \prime} \mathrm{O}$ \\
\hline 59 & Acacia heterophylla & Acace & $5^{\circ} 12 ’ 1.58 ’ ’ \mathrm{~S}$ & $37^{\circ} 19^{\prime} 60.97^{\prime \prime} \mathrm{O}$ \\
\hline 60 & Azadirachta indica & Neem & $5^{\circ} 12^{\prime} 1.70^{\prime \prime} \mathrm{S}$ & $37^{\circ} 19^{\prime} 61.59^{\prime \prime} \mathrm{O}$ \\
\hline 61 & Azadirachta indica & Neem & $5^{\circ} 12^{\prime} 1.20^{\prime \prime} \mathrm{S}$ & $37^{\circ} 19^{\prime} 57.97^{\prime \prime} \mathrm{O}$ \\
\hline 62 & Azadirachta indica & Neem & $5^{\circ} 12^{\prime} 1.27^{\prime \prime} \mathrm{S}$ & $37^{\circ} 19^{\prime} 58.53^{\prime \prime} \mathrm{O}$ \\
\hline 63 & Mangifera indica & Mangueira & $5^{\circ} 12^{\prime} 1.82{ }^{\prime \prime} \mathrm{S}$ & $37^{\circ} 19^{\prime} 57.56^{\prime \prime} \mathrm{O}$ \\
\hline 64 & Prosopis juliflora & Algaroba & $5^{\circ} 12^{\prime} 2.08^{\prime \prime} \mathrm{S}$ & $37^{\circ} 19^{\prime} 54.64^{\prime \prime} \mathrm{O}$ \\
\hline 65 & Terminalia catappa & Castanhola & $5^{\circ} 12 ’ 2.10^{\prime \prime} \mathrm{S}$ & $37^{\circ} 19^{\prime} 60.77^{\prime \prime} \mathrm{O}$ \\
\hline 66 & Eucalyptus globulus & Eucalipto & $5^{\circ} 12^{\prime} 2.13^{\prime \prime} \mathrm{S}$ & $37^{\circ} 19^{\prime} 65.10^{\prime \prime} \mathrm{O}$ \\
\hline 67 & Azadirachta indica & Neem & $5^{\circ} 12^{\prime} 2.09^{\prime \prime} \mathrm{S}$ & $37^{\circ} 19^{\prime} 64.55^{\prime \prime} \mathrm{O}$ \\
\hline 68 & Acacia heterophylla & Acace & $5^{\circ} 12^{\prime} 2.26^{\prime \prime} \mathrm{S}$ & $37^{\circ} 19^{\prime} 58.87^{\prime} \mathrm{O}$ \\
\hline 69 & Bromelia L. & Bromélia & $5^{\circ} 12^{\prime} 2.54^{\prime \prime} \mathrm{S}$ & $37^{\circ} 19^{\prime} 57.59^{\prime \prime} \mathrm{O}$ \\
\hline 70 & Tabebuia caraíba & Craibeira & $5^{\circ} 12^{\prime} 2.53^{\prime \prime} \mathrm{S}$ & $37^{\circ} 19^{\prime} 57.35^{\prime \prime} \mathrm{O}$ \\
\hline 71 & Azadirachta indica & Neem & $5^{\circ} 12^{\prime} 3.43^{\prime \prime} \mathrm{S}$ & $37^{\circ} 19^{\prime} 63.23^{\prime \prime} \mathrm{O}$ \\
\hline
\end{tabular}

Dentre todas as espécies amostradas, 24 espécies $(25,3 \%)$ são representantes de espécies nativas e 71 espécies $(74,7 \%)$ são exóticas. Segundo estes resultados, a arborização da UFERSA segue o padrão observado na maioria das áreas verdes públicas das cidades brasileiras, com grande número de espécies exóticas em relação às nativas do Brasil (LORENZI, 1992).
Os resultados obtidos demonstram que a arborização da UFERSA seguiu a tendência do cultivo de espécies exóticas, provavelmente por questões históricas (MILANO; DALCIN, 2000), e pela falta de informação existente acerca da produção de mudas, potencial econômico, ornamental e paisagístico, e ainda pela dificuldade de encontrar mudas e sementes de espécies nativas no mercado (MENDONÇA, 2004).

Figura 1. Pontos referentes às espécies vegetais nativas (azuis) e exóticas (vermelhos). Mossoró- RN, 2014.

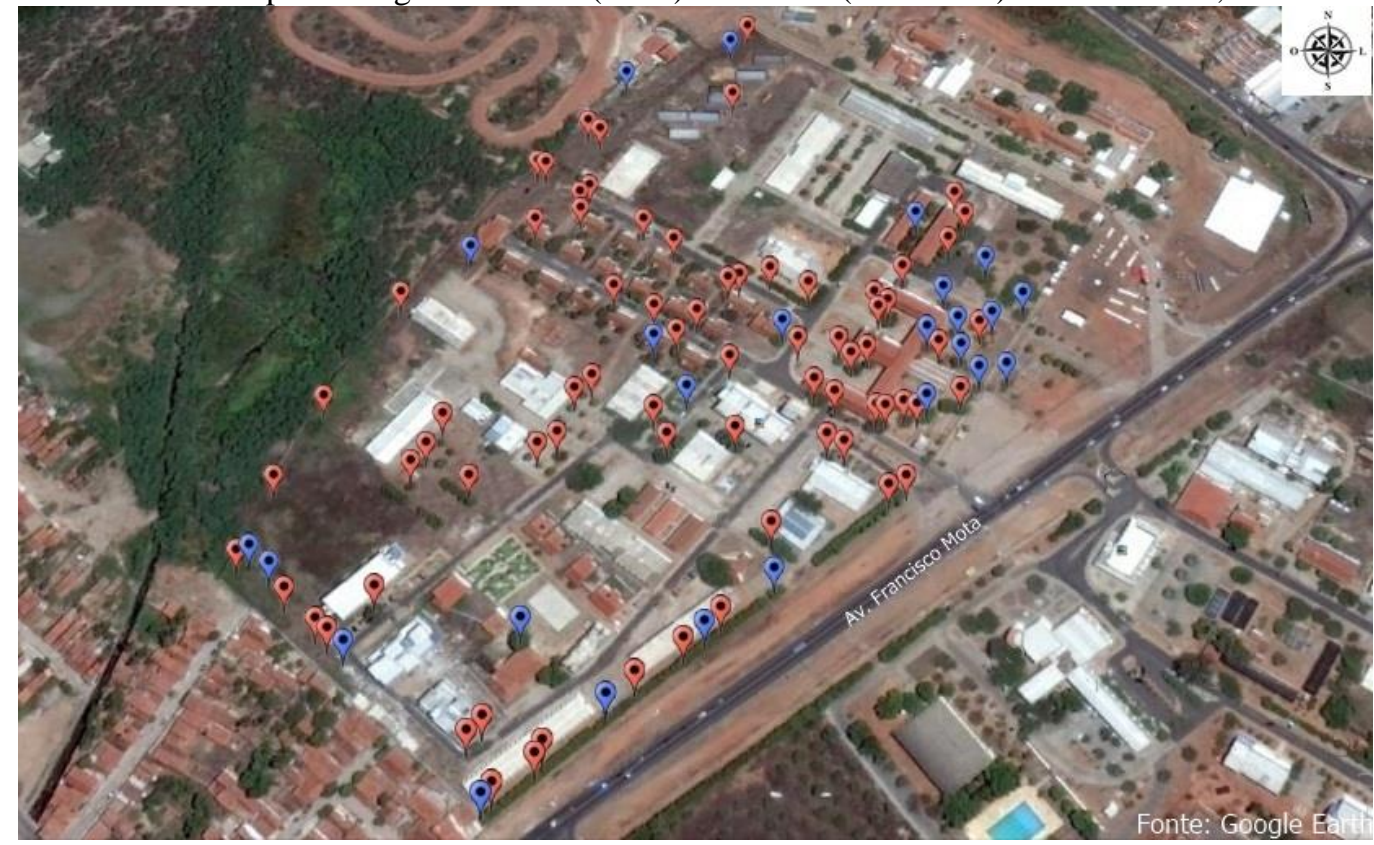




\section{CONCLUSÕES}

Em vista de uma melhor estrutura física fica evidente a necessidade de uma maior arborização, tendo como incentivo o plantio de espécies nativas, que proporcionem sombra, alimento e sirvam de abrigo para a fauna local, não apenas na UFERSA, mas em toda a região que vem perdendo sua flora natural em favor de plantas exóticas.

\section{REFERÊNCIAS BIBLIOGRÁFICAS}

ARVÓRES BRASIL. Porque Não Plantas Exóticas. Disponível em: $<$ http://www.arvoresbrasil.com.br/?pg=arvore_porque_na o_exoticas > Acesso em: 07 Fev. 2016.

BARBOSA, F.; COSTA, A. M. de B.; SILVA, F. M. Cooperativa Carnaúba Viva: preservação e valorização da caatinga para o desenvolvimento sustentável do semiárido brasileiro. Sociedade e Território, Natal, v. 21, no 1 - 2 (Edição Especial), (p. 72, 2009a).

CARMO FILHO, F.; ESPÍNOLA SOBRINHO, J.; MAIA NETO, J. M. Dados meteorológicos de Mossoró (janeiro de 1989 a dezembro de 1990), Mossoró: ESAM, FGD, 1991, 110p, Coleção Mossoroense, Série C, 630.

CORADIN, L.; SIMINSKI A.; REIS, A. Espécies nativas da flora brasileira de valor econômico atual ou potencial: Plantas para o futuro - Região Sul. Brasília: MMA, 2011. 934p.

GISP - Programa Global de Espécies Invasoras. 2005. América do Sul invadida. A crescente ameaça das espécies exóticas invasoras. 80p.

IBGE. Cidades: Mossoró. Brasília: IBGE, 2014. Disponível em: <http://cidades.ibge.gov.br> Acesso em: 16 Jan. 2016.

INPE - INSTITUTO NACIONAL DE PESQUISAS ESPACIAIS. Projeto Prodes - Monitoramento da floresta amazônica brasileira por satélite. Disponível em: <http://www.obt.inpe.br/prodes/index.php> Acesso em: 05 Fev. 2016.

INSTITUTO HÓRUS. 2007. Espécies exóticas invasoras: Fichas técnicas. Disponível em: <www.institutohorus.org.br/> Acesso em 05 Fev. 2016.

LORENZI, H. Árvores brasileiras: manual de identificação e cultivo de plantas arbóreas nativas do Brasil. Nova Odessa: Plantarum, 1992. 352 p.

MENDONÇA F. B. 2004. Árvores do campus: levantamento florístico das angiospermas arbóreas da Cidade Universitária "Armando Salles de Oliveira". Universidade de São Paulo, São Paulo, MSc. diss.
MILANO, M.; DALCIN, E. Arborização de vias públicas. Rio de Janeiro: LIGHT, 2000. 226 p.

OLIVEIRA, R. E.; ZAKIA, M. J. B. Guia para análise de viveiros de mudas nativas. Check-list para verificação da adequação legal, socioambiental e ecológica de viveiros de mudas florestais. IPEF, 2010.

RANDALL, R. P. 2002: A global compendium of weeds. Melbourne: R. G. and F. J. Richardson

SILVA, G. O ambientalismo tardio: a Amazônia como temática ambiental no jornalismo impresso paulista. 2006. Dissertação (Mestrado em Comunicação) Faculdade Cásper Líbero, São Paulo, 2006. 